\title{
Investigating the Effects of picture book on Reducing Distress Caused by Intravenous Catheterization among School-aged Children (randomized clinical trial)
}

Masoumeh Kanikzadeh

BPUMS

Rahim Tahmasebi

BPUMS

farideh bahreini

BPUMS.AC.IR

faezeh jahanpour ( $\nabla$ f.jahanpour@bpums.ac.ir)

BPUMS https://orcid.org/0000-0001-6626-6184

Research article

Keywords: anxiety, pediatric, Intravenous Catheterization, education, picture book

Posted Date: June 8th, 2020

DOI: https://doi.org/10.21203/rs.3.rs-19315/v2

License: (1) (1) This work is licensed under a Creative Commons Attribution 4.0 International License.

Read Full License 


\section{Abstract}

Background: Hospitalized pediatric patients experience pain, fear, and distress during intravenous catheterization. This study investigates the effects of a picture book on reducing distress caused by intravenous (IV) catheterization among school-aged children.

Methods: seventy four Pediatric emergency room patients age 6-12 years old requiring Iv Infusion were enrolled in this randomized clinical trial. The intervention, providing a description of the IV catheterization procedure using a picture book, was compared to the same description of the procedure without a picture book (standard of care). Subject distress was measured using the Observation Scale of Behavior Distress Revised (OSBD_R) which assigns a score to eight observable behaviors. Before, during, and after the procedure at 15-second intervals (measured using a timer), a trained observer assigned an OSBD_R score of distress. A total score of 22 is considered maximum distress. The primary endpoint for the study was OBSD_R score difference in Phase 3 (Performing catheterization procedure, attaching and infusion therapy). Chi-square, Mann-Whitney and Wilcoxon tests were used for data analysis.

Results: The mean distress score in third phases for the intervention group $(0.667 \pm 0.610)$ was significantly less than that of the control group (3.218 \pm 1.799$)$. A significant difference in distress score was observed before and after the intervention $(p<0.001)$.

Conclusions: Children's distress due to IV catheterization can be reduced with a simple, nonpharmacological picture book intervention. The children's orientation on the IV catheterization plays a significant role in controlling and reducing their distress during and after the intervention. We suggested using the picture book to reduce the children distress before Intravenous Catheterization.

\section{Background}

Injections provoke pain, fear, and distress among hospitalized children (1). Taddio et al. (2012) reported that $24 \%$ of mothers and $63 \%$ of children are afraid of needles (2). Intravenous catheters are a common invasive medical procedure required for a variety of therapeutic interventions (3). In addition to causing pain, this procedure provokes distress in children, their parents, and even nurses (4). In Iran, Borhani et al. (2012) reported that children experience mild or severe pain and anexiety during IV catheterization. In addition, they reported a significant relationship between post-injection pain and pre-injection anxiety (5). Over-distress can lead to a child's unwillingness to receive medical treatment resulting in negative feelings towards the personnel and preventing effective medical treatment (6).

Various pharmacologic and non-pharmacologic approaches have been adopted to reduce children's pain and distress during medical procedures (7). High level of pain and distress were reported in children even with pharmacological interventions (8).Preference for non-pharmacologic approaches for reducing pain and distress have increased as their efficacy, relative safety, and feasibility are appreciated. Examples of non-pharmacologic interventions during IV catheterization placement in children include orientation, behavioral distractions, tactile interventions, sweet solutions for infants, and cold analgesia $(9,10)$. 
Focusing on orientation, interventions can include providing instructions on future procedures, studying picture books on similar experiences, and introducing videos (11). Children's illustrated books provide instructions using brief, fun, colorful, and readable material (12). Picture books can help children identify the context of the medical procedure and build their own version of the story.

Nurses who take the patient's distress seriously and try to alleviate it can establish a better therapeutic relationship and increase the patient and their parent's satisfaction levels (13). No study, to the best of our knowledge, has been reported regarding distress reduction in school-aged children using orientation to IV catheterization through picture books in a pediatric emergency room (ER). We hypothesized that the use of a picture book will significantly reduce distress levels in children undergoing IV catheterization, when compared with standard of care in the pediatric emergency room (ER). Thus, the current study of pediatric ER patients evaluates the effects of IV catheterization orientation through picture book on school-aged children's distress.

\section{Methods}

This randomized clinical trial study was conducted at Shohadaye Khalij Fars Hospital in Bushehr -Iran from (7/30/2017) to $(11 / 11 / 2017)$ Children age 6-12 who presented to the pediatric ER and required IV catheterization for infusion were randomized to intervention or standard of care. Inclusion criteria included full consciousness, the parent's informed consent, verbal assent of the pediatric patient, and presence of a parent during the study. Exclusion criteria included taking pain and distress relief drugs (at least 6 hours before the procedure), presence of hearing, verbal, mental, or visual limitations that may interfere with picture book use, and any psychological distress requiring emergency IV therapy. Any patient who was randomized to the trial and had an unsuccessful initial IV catheterization was excluded and their data was not used in the final analysis.

\section{Sample size:}

To ensure sufficient power to detect differences between the groups, power analysis was used to estimate the sample size. We referenced a previous study (14) to predict a medium effect size for the intervention (effect size:0.84). To predict this effect size at a $5 \%$ significance level $(p<0.05)$ and power of 0.90 , we calculated that 32 subjects would be required in each group, We allowed for an attrition rate of $10 \%$ giving a total sample size of 74 .

This trial was approved by the institutional ethics committee, and written informed consent was obtained from all parents, along with verbal assent of all pediatric study subjects. The trial was registered with the Iranian Registry of Clinical Trials (IRCT) (code: IRCT2017041712830N22) on May 29, 2017.

\section{Development of the Intervention:}

The picture book intervention was developed by a graphic design postgraduate student and professors of Nursing and Midwifery at Bushehr University of Medical Sciences. This book contained six child-friendly 
drawings in which peripheral IV catheterization and infusion therapy are demonstrated in a step-by-step manner.

The main goal of this picture book was to orientate subjects to the steps, equipment, and expectations of IV catheterization and infusion.

\section{Procedure}

First, subjects were randomized using the convenience method. Then, they were randomly assigned into intervention or control groups through drawing from a collection of 74 cards (by the nurse) labeled from 1 to 74 after the parents and their children provided informed consent and verbal assent, respectively. The cards with even numbers were assigned to the control group (standard of care) and odd numbers were assigned to the intervention group. In the intervention group $(n=36)$, one study subject and in the control group ( $n=38$ ) three subjects were Discontinued due to unsuccessful initial catheterization (Refer to CONSORT FLOW CHART - figure2). Demographic information was provided by one of the parents. Once the patient received orders for Infusion requiring IV catheterization, the subject and a parent were brought to a treatment. The IV catheterization was performed by an experienced nurse. Research nursing students were trained on standardized OSBD_R scoring. Standard care in both groups for pain included verbal explanations, caressing, and presence of parents for support. Although school-aged children can tolerate parents' absence, hospitalization may enhance their needs for security and parental presence (15). The

procedure was divided into 4 phases. During the first phase, the nurse introduced themselves to the child and parent while preparing the intravenous IV catheterization equipment for 3 minutes. Then, between phases 1 to phases 2 the nurse oriented the subject to IV catheterization and infusion therapy by using the picture book. The control group received the same orientation without the picture book. Between phase 1 and 2, the nurse assessor the OSBD_R scorer left the treatment room; ultimately thought the scorer was not blinded as they could hear the treatment room proceedings. Phase 2 was considered the interval of time required for vein identification and preparation. Phase 3 included the entire IV catheterization procedure. Finally, phase 4 was the 3 minutes immediately following completion of the IV catheterization. No other pain interventions occurred in the either group. Due to the nature of the study, parents, nurses, and participants were not blinded.

\section{Evaluation method}

Distress was measured by Observation Scale of Behavior Distress_ Revised (OSBD_R), a standard scale to measure distress validated by Elliot et al $(18)$ and utilized in in similar studies $(12,16)$. Specifically for an Iranian population, Ghazavi et al. confirmed the reliability of this scale $(r=0.70)(17)$. The scale consists of eight behaviors indicating children distress resulting from therapeutic procedures. The behaviors and their scores include crying (1.5), screaming (4.0), restraining (4.0), and verbal resistance (2.5), and information-seeking (1.5), solicitation of emotional support (2.0), verbal pain expression (2.5) and flailing (4.0). A total score of 22 is considered the highest level of distress (17). Further, demographic information such as age, gender, birth order, parents' education level, and residential area was collected. 
The presence or absence of these eight operationally -defined behaviors were recorded by trained nurse observer (assessor) at 15-second intervals during the four phases.

The summed scores were summed during each 15-second interval and divided by the number of intervals to obtain a mean score for each phase. The phases are summarized in Figure 3.

\section{Data analysis:}

The data were analyzed by SPSS software (version 22). The Kolmogorov-Smirnov and Shapiro-Wilk tests were used to check the normal distribution of distress data. The OSBD_R scores were not normally. distributed. Therefore, we used the Friedman test, a non-parametric test equivalent of the repeated measure analysis of variance, to examine changes in distress intensity over time.

In addition to descriptive statistics, Chi-square analysis compared the distribution of qualitative demographic variables between the two groups before intervention. The Mann-Whitney tests were used for comparing the mean (median) distress score between the two groups in each phase (?), and Friedman tests were used for evaluating changes of mean (median) distress score duration of intervention in intervention and control group respectively. The significance level was set at $p<0.05$ in all tests.

\section{Results}

A total of 74 subjects were randomized for trial participation. Due to unsuccessful initial peripheral IV catheterization IV, three children from the control group and one from the intervention group were excluded. 70 subjects were eligible for analysis (Figure 1, Consort flow diagram). Based on the results, no significant difference was observed between the intervention and control groups in terms of demographic variables such as gender, age, birth order, parents' education level, the parent accompanying the child, and residential area. In other words, the two groups were homogenous regarding demographic features. the mean age of intervention and control groups was $8.26 \pm 2.147$ and $8 \pm 2.072$, respectively. Demographic information and their comparison in the intervention and control groups can be seen in (Table 1).

The distress scores of both groups in the first phase (before intervention) were not significantly different. Further, the mean distress scores of the intervention group $(0.667 \pm 0.610)$ after intervention were significantly less than those of the control group (3.218 \pm 1.799$)$. After intervention, the picture book group had considerably less distress in the second, third, and fourth phases, compared to the control group. Results in the phases after intervention were significantly different in both groups (Table 2). The difference decreased the distress level in the intervention group while it increased the distress level in the control group.

\section{Discussion}


In the present study, a picture book on IV catheterization and infusion was created and the behavioral distress of school-aged children in the ER was evaluated. Based on the significant difference in distress scores after study intervention, we believe this kind of intervention provides an opportunity for nurses to decrease school-aged children's fears in facing IV catheterization. In addition, this study suggests children's distress can be reduced through orientation to therapeutic intervention.

This is in line with other nonpharmacological interventions which have shown reduced distress when applied just prior to or during potentially distressing therapeutic interventions. For example, a 2015 study concluded that interactive computer games controlled the injection distress during and after the game among the children in the intervention group effectively (1).

It was reported in a study performed in 2013 that the distress level of patients undergoing operation, after implementation of blended education program (in forms of verbal explanation, educational videos and pamphlet) was significantly lower than that of the control group. However, distress level in the second test (releasing time) was not significantly different in both groups (18), maybe due to the lengthy interval between the education and the second post-test. Furthermore, the method, age-range and the performed procedure on the patients were different from the present study.

In this regard a study published in 2013 concluded that children's storybooks containing operation related issues are effective for reducing the distress before the operation among 5 to 11 year-old children, especially 7-year-old girls (19). It has been suggested that children orientation about the injection procedures through games can considerably reduce their distress during the procedure (20). In addition, in 2015 it was reported in study that distress decreased considerably among children who underwent interventions through reading manual and role-playing the peripheral cannulation on a teddy bear (21). These results are consistent with the findings of the present study.

According to Langford, 6 to 12 year-old children acquire logical thinking and their distress could be reduced realistically through explanation and briefing before the procedure (22). A similar study in preschool age children did not find clear benefit of a picture book on reducing distress levels during IV catheterization (12). We hypothesized that school aged children 6-12 would benefit from the picture book orientation given this advanced development. Our study findings support this hypothesis in finding reduced distress levels in older children with use of the picture book orientation.

Thus, nurses can play an important role within the framework of the holistic, patient-centered and individualized health care.

\section{Conclusions}

Orientation to IV catheterization through picture books can reduce the distress among school-aged children during and after performing the treatment in the intervention group. Therefore, it is recommended that picture book should be routinely used for children during IV catheterization in order to decrease their 
distress. However, further studies with samples in different ages and cultural groups are warranted to provide conclusive evidence.

\section{Limitations Of The Study}

\section{Limitation of the study}

The following Items are considered limitations of the present study, which may affect children's distress level or reliability.

1-Variable the duration of IV catheterization preparation and procedure in Phase 2 and 3; it is possible that prolonged procedure time can affect level of distress.

2- ER crowding and louder environmental noise may affect a child's focus on the intervention 3- Cultural, educational and parenting style differences between families can affect children's possible reactions to pain and distress.

4- Inter-rater reliability was not captured due to concern for due to the increasing the child's distress of having two observer OBSD_R scorers in the treatment at the same time.

5- Despite attempts to achieve blinding, the OSBD_R scorer was not blinded to the randomization assignment.

6- The trial excludes children under 6 years old and over 12 and any results will not be generalizable outside of this population

7- Distress levels were not obtained via child self-report (to prevent interruption of the orientation the child was allocated to receive)

\section{Strengths of the study:}

Various actions undertaken in order maximize the validity and reliability of this trial includes:

1- Standardized study documents (clinical research data gathering forms, informed consent forms) were standardized;

2- The OSBD_R scoring nurse was trained on gathering the data to ensure quick and accurate distress scale scoring.

3-using the comprehensive tool scale for gathering data consists of eight behaviors (verbal and nonverbal).

\section{Implications of results:}


This research is applicable for nursing practice in all pediatric wards and pediatric emergency rooms in hospitals. This kind of nonpharmacological intervention can provide an opportunity for nurses to reduce distress in school-aged children requiring intravenous catheterization. This allows the opportunity to build a therapeutic relationship with a relatively safe, simple intervention.

\section{Declarations}

\section{Ethics approval and consent to participate:}

The present research was approved by the Ethical Committee of Bushehr University of Medical Sciences and was registered at Iranian Registry of Clinical Trials (IRCT) (code:

IRCT2017041712830N22). Registered in 2017-05-29. After obtaining permission from Research Deputy of Bushehr University of Medical Sciences, presenting the introductory letter to the Research Center, introducing the authors of this paper to the Research Units and explaining the advantages of reading and safe nature of the intervention to the parents, they were given the option to participate in this study. The study was executed in pediatric emergency ward of Shohadaye Khalij Fars Hospital in Bushehr. The parents' informed consent was taken in written form and the children gave an oral agreement. The participants were assured about the confidentiality of the collected data.

\section{Consent for publication:}

'Not applicable'

\section{Availability of data and material:}

The data are available from the corresponding author on reasonable request.

\section{Competing interests:}

No conflict of interest has been declared by the authors.

\section{Funding:}

The research funded by Research Deputy of Bushehr University of Medical Sciences. The funder provided review, advice, support and monitored study progress but did not have a role in study design or data collection, analysis and interpretation.

\section{Authors' contributions:}

M K: writing the initial protocol, the manuscript draft and collecting the data

$R T$ : provided study design, statistical and methodological expertise 
F B:collecting the data

F J: (corresponding author): develop the protocol, project management; review the research proposal and the manuscript.

"All authors approved the final version of the manuscript."

\section{Corresponding author:}

Correspondence to: Faezeh Jahanpour

\section{Acknowledgements}

The present study was based on a MSc. Thesis of pediatric nursing. The authors would like to thank and appreciate the Deputy of Research Center at Bushehr University of Medical Sciences for funding this research. In addition, the authors hereby would like to thank the directors of Shohadaye Khalij Fars Hospital, the head nurse and nurses at pediatric emergency wards for their kind contribution to this research.

\section{Abbreviations}

IV: intravenous

ER: emergency room

\section{References}

1. Ebrahimpour F, Sadeghi N, Najafi M, Iraj B, Shahrokhi A. Effect of playing interactive computer game on distress of insulin injection among type 1 diabetic children. Iranian journal of pediatrics. 2015;25(3).

2. Taddio A, Ipp M, Thivakaran S, Jamal A, Parikh C, Smart S, et al. Survey of the prevalence of immunization non-compliance due to needle fears in children and adults. Vaccine. 2012;30(32):4807-12.

3. Khalili Shomia S, Safavi M, Yahyavi SH, Farahani H. Assessment of the Effect of EMLA Cream on Vein Puncture Pain Severity with Vein Catheter in the Patients Undergoing Cesarean Section: Randomized Placebo Controlled Trial. Journal of Mazandaran University of Medical Sciences. 2012;22(91):82-8.

4. Martin HA. The Power of Topical Anesthetics and Distraction for Peripheral Intravenous Catheter Placement in the Pediatric Perianesthesia Area. Journal of PeriAnesthesia Nursing. 2017:1-7.

5. Borhani F, Bagherian S, Abaszadeh A, Ranjbar H, Tehrani H, Soleimanizadeh L. Correlation between anxiety and pain due to intravenous catheters in children with thalassemia. Scientific Journal of Iranian Blood Transfusion Organization. 2012;9(2):170-9. 
6. Li WHC, Chung JOK, Ho KY, Kwok BMC. Play interventions to reduce anxiety and negative emotions in hospitalized children. BMC Pediatrics. 2016;16(1):1-9.

7. Inal S, Kelleci M. Distracting children during blood draw: Looking through distraction cards is effective in pain relief of children during blood draw. International Journal of Nursing Practice. 2012;18(2):210-9.

8. Wong CL, Lui MMW, Choi KC. Effects of immersive virtual reality intervention on pain and anxiety among pediatric patients undergoing venipuncture: a study protocol for a randomized controlled trial. Trials. 2019;20(1):369.

9. Redfern RE, Chen JT, Sibrel S. Effects of thermomechanical stimulation during vaccination on anxiety, pain, and satisfaction in pediatric patients: A randomized controlled trial. Journal of Pediatric Nursing: Nursing Care of Children and Families. 2018;38:1-7.

10. Talebi s, Ganjloo J, Rakhshani M, Asghari Nekah S. Comparison the effect of orientation program used by visual concept map and face to face method on fear and anxiety of children. Paediatrics Nursing. 2015;4:32-44.

11. Lerwick JL, editor Psychosocial implications of pediatric surgical hospitalization. Seminars in pediatric surgery; 2013: Elsevier.

12. Tsao Y, Kuo HC, Lee HC, Yiin SJ. Developing a medical picture book for reducing venipuncture distress in preschool-aged children. International journal of nursing practice. 2017;23(5).

13. Toobaee E. Investigating the role of press illustration and its impact on promoting the publishing industry of Iran. Analytical-Research Quarterly Journal of Ketab-e-Mehr. 1393;11,12:136-52.

14. Bagherian S, Borhani F, Zadeh AA, Ranjbar H, Solaimani F. The effect of distraction by bubble-making on the procedural anxiety of injection in Thalassemic school-age children in Kerman Thalasemia center. Advances in Nursing \& Midwifery. 2012;21(76):52-9.

15. Reyhani T, Dehghan Z, Shojaeian R, Ashgharinekah S, Behnam Vashani H. The influence of the puppet Kolah Ghermezi on preoperative anxiety among hospitalized children with appendicitis in $\mathrm{Dr}$ Shaikh hospital of Mashhad. Evidence Based Care. 2014;4(3):77-86.

16. Caprilli S, Anastasi F, Grotto RPL, Abeti MS, Messeri A. Interactive music as a treatment for pain and stress in children during venipuncture: a randomized prospective study. Journal of Developmental \& Behavioral Pediatrics. 2007;28(5):399-403.

17. Ghazavi A, Pouraboli B, Sabzevari S, Mirzai M. The effect of foot reflexology massage on vital signs and anxiety related to injection during chemotherapy in children. Medical-Surgical Nursing Journal. 2016;4(4):56.

18. Tol A, Pardel Shahri M, Esmaelee Shahmirzadi S, Mohebbi B, Javadinia S. Effect of blended education program on anxiety among orthopedic patients surgery. Journal of nursing education. 2013;2(3):1-8.

19. Tunney AM, Boore J. The effectiveness of a storybook in lessening anxiety in children undergoing tonsillectomy and adenoidectomy in Northern Ireland. Issues in comprehensive pediatric nursing. 2013;36(4):319-35. 
20. Hamed Tavassoli S, Alhani F, Hajizadeh E. Investigating of the effect of familiarzation play on the injective procedural anxiety in B-Thalassemic pre-school children. Journal of Urmia Nursing And Midwifery Faculty. 2012;7(26):42-9.

21. Bassampour $S$. The effect of preoperative education on pre and post surgery anxiety level in patients undergoing open heart surgery. Quarterly Journal of Payesh. 1383;3(2):139-44.

22. Langford R. The preparation of children for surgery anaesthesia tutorial of the week 132. 2009.

\section{Tables}

Distribution of qualitative demographic variables among the participantsTable 1.

\begin{tabular}{|c|c|c|c|c|c|}
\hline \multicolumn{2}{|c|}{ Demographic Features } & \multicolumn{2}{|c|}{ Number (\%) } & \multirow[t]{2}{*}{$\chi^{2}$} & \multirow[t]{2}{*}{ P value } \\
\hline & & $\begin{array}{l}\text { Intervention } \\
\text { Group }(\mathrm{n}=35)\end{array}$ & $\begin{array}{c}\text { Control } \\
\text { Group }(n=35)\end{array}$ & & \\
\hline Gender & Girl & $20(57.1 \%)$ & $17(48.6 \%)$ & 0.516 & 0.473 \\
\hline \multirow{3}{*}{ Birth Order } & 1 & $16(45.7 \%)$ & $22(62.9 \%)$ & \multirow[t]{3}{*}{2.090} & \multirow[t]{3}{*}{0.352} \\
\hline & 2 & $15(42.9 \%)$ & $10(28.6 \%)$ & & \\
\hline & 3 and Above & $4(11.4 \%)$ & $3(8.6 \%)$ & & \\
\hline \multirow{3}{*}{$\begin{array}{l}\text { Father's } \\
\text { Education }\end{array}$} & Under Diploma & $10(28.6 \%)$ & $7(20 \%)$ & \multirow[t]{3}{*}{0.840} & \multirow[t]{3}{*}{0.657} \\
\hline & Diploma & $13(37.1 \%)$ & $16(45.7 \%)$ & & \\
\hline & Academic & $12(34.3 \%)$ & $12(34.3 \%)$ & & \\
\hline \multirow[t]{3}{*}{ Mother's Education } & Under Diploma & $13(37.1 \%)$ & $10(28.6 \%)$ & \multirow[t]{3}{*}{0.702} & \multirow[t]{3}{*}{0.704} \\
\hline & Diploma & $13(37.1 \%)$ & $16(45.7 \%)$ & & \\
\hline & Academic & $9(25.7 \%)$ & $9(25.7 \%)$ & & \\
\hline \multirow[t]{2}{*}{ Accompanying Parent } & Father & $11(31.4)$ & $12(34.3 \%)$ & \multirow[t]{2}{*}{0.065} & \multirow[t]{2}{*}{0.799} \\
\hline & Mother & $24(68.6 \%)$ & $23(65.7 \%)$ & & \\
\hline \multirow[t]{2}{*}{ Residential Area } & City & $33(94.3 \%)$ & $31(88.6 \%)$ & \multirow[t]{2}{*}{0.729} & \multirow[t]{2}{*}{0.393} \\
\hline & Village & $2(5.7 \%)$ & $4(11.4 \%)$ & & \\
\hline
\end{tabular}


Children's mean (Median) distress score during four phasesTable 2.

\begin{tabular}{|c|c|c|c|c|}
\hline Groups & Intervention $(\mathrm{n}=35)$ & Control $(\mathrm{n}=35)$ & \multirow[t]{2}{*}{ Mann-Whitney Test } & \multirow[t]{2}{*}{$\mathrm{P}$ value } \\
\hline Phases & $\begin{array}{c}\text { mean } \pm \text { SD } \\
\text { (Median) }\end{array}$ & $\begin{array}{c}\text { mean } \pm \text { SD } \\
\text { (Median) }\end{array}$ & & \\
\hline 1 & $\begin{array}{c}0.844 \pm 0.901 \\
(0.625)\end{array}$ & $\begin{array}{c}0.724 \pm 1.015 \\
(0.250)\end{array}$ & 546.000 & 0.417 \\
\hline 2 & $\begin{array}{c}0.675 \pm 0.979 \\
(0.428)\end{array}$ & $\begin{array}{c}2.197 \pm 1.906 \\
(1.590)\end{array}$ & 278.500 & $<0.001$ \\
\hline 3 & $\begin{array}{c}0.667 \pm 0.610 \\
(0.550)\end{array}$ & $\begin{array}{c}3.218 \pm 1.799 \\
(2.800)\end{array}$ & 78.500 & $<0.001$ \\
\hline 4 & $\begin{array}{c}0.032 \pm 0.875 \\
(0.000)\end{array}$ & $\begin{array}{c}0.303 \pm 0.819 \\
(0.000)\end{array}$ & 456.000 & 0.017 \\
\hline $\begin{array}{c}\text { Friedman Test } \chi^{2} \\
(p \text { value })\end{array}$ & $\begin{array}{c}35.245 \\
(<0.001)\end{array}$ & $\begin{array}{c}63.776 \\
(<0.001)\end{array}$ & - & \\
\hline
\end{tabular}

\section{Figures}

(1) Why is this procedure required?

(2) What will happen during the procedure?

(3) Equipment and tools required for procedure

\section{Figure 1}

The content of the picture book 


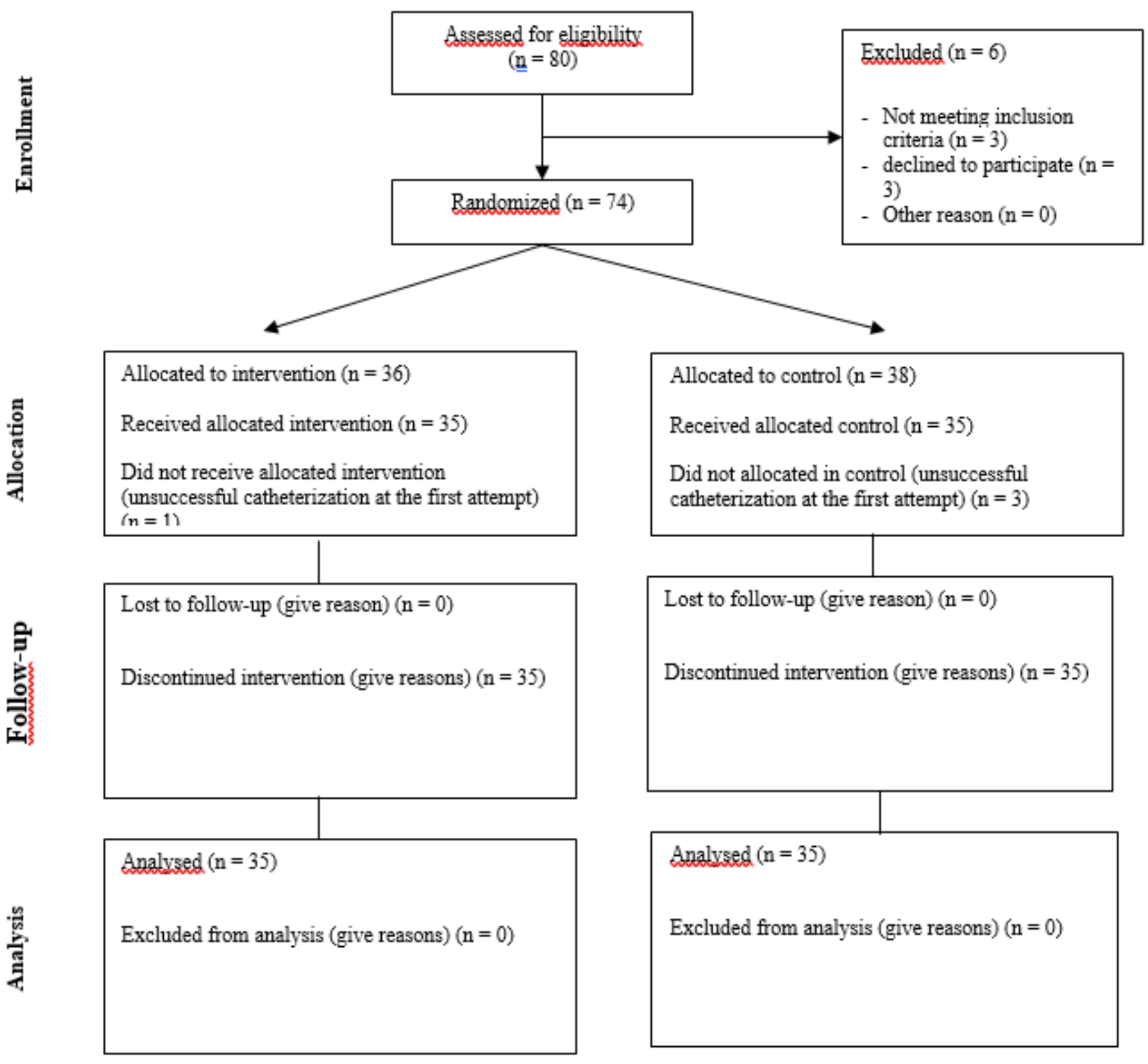

\section{Figure 2}

Consort flow chart 
These phases include the following features in the intervention and control groups:

Phase 1: In the treatment room for three minutes

Phase 2: Finding the injection spot and preparing the skin

Phase 3: Performing catheterization procedure, attaching and infusion therapy

Phase 4: From the end of phase 3 to 3 minutes after IV placement

\section{Figure 3}

The phases of the tool

\section{Supplementary Files}

This is a list of supplementary files associated with this preprint. Click to download.

- supplement1.docx

- supplement2.docx

- CONSORTExtensionNPT2017FlowDiagram.doc 